УДК 616.3-006.52-072.1

https://doi.org/10.18692/1810-4800-2020-1-105-109

\author{
Инвертированная папимома кминовидной пазухи \\ М. А. ЭАже ${ }^{1}$, А. Ю. Овчинников ${ }^{1}$, А. Ю. Щербаков ${ }^{1}$ \\ 1 Московский государственный медико-стоматологический университет имени А. И. ЕвАокимова, \\ Москва, 127473, Россия
}

\title{
Inverted papilloma of sphenoid sinus
}

\author{
M. A. Edzhe ${ }^{1}$, A. Yu. Ovchinnikov ${ }^{1}$, A. Yu. Shcherbakov ${ }^{1}$ \\ ${ }^{1}$ Evdokimov Moscow State University of Medicine and Dentistry, \\ Moscow, 127473, Russia
}

\begin{abstract}
Инвертированная папиллома или переходно-клеточная папиллома полости носа и околоносовых пазух - это доброкачественная опухоль, составляющая 0,4-7\% всех новообразований полости носа и околоносовых пазух. Изолированное поражение одной околоносовой пазухи является редким и отмечается в 5\% наблюдений. Больная Т., 57 лет, с жалобами на нарушение носового дыхания справа, постоянную заложенность правой половины носа, периодическую заложенность правого уха и головную боль на стороне поражения поступила на плановое хирургическое лечение в оториноларингологическое отделение Клинического медицинского центра МГМСУ им. А. И. Евдокимова. Перечисленные симптомы отмечает на протяжении 10 лет. На компьютерной томограмме головы выявлено субтотальное затемнение правой основной пазухи с распространением в полость носа и носоглотку. Предварительно выполнена биопсия опухоли с последующим гистологическим исследованием, в результате которого установлена инвертированная переходно-клеточная папиллома. Выполнены трансназальный эндоскопический подход к задним отделам полости носа с удалением всей передней и части нижней стенок правой клиновидной пазухи, удаление опухоли и широкая диссекция слизистой оболочки задних отделов полости носа и правой клиновидной пазухи в целях удаления всех возможных локусов первичного очага. Планирование трансназальной операции начинается с установления клинического диагноза, подтвержденного в сомнительных случаях результатами гистологического исследования, анализа результатов необходимого метода визуализации и выбора адекватного доступа, способствующего полноценному воздействию на патологическое образование и проведению безопасной контролируемой хирургии в аспектах кровопотери и повреждения критических сосудисто-нервных структур при наименьших из возможных временных затратах.

Ключевые слова: инвертированная папиллома, основная пазуха, трансназальный эндоскопический подход.
\end{abstract}

Для цитирования: Эдже М. А., Овчинников А. Ю., Щербаков А. Ю. Инвертированная папиллома клиновидной пазухи. Российская оториноларингология. 2020;19(1):105-109. https://doi.org/10.18692/18104800-2020-1-105-109

Inverted papilloma of the nasal cavity and paranasal sinuses is a benign tumor, accounting for approximately 0.4 to $7 \%$ of all sinonasal neoplasms. An isolated lesion of one paranasal sinus is a rare disease, it occurs in $5 \%$ of cases. Female patient T., 57 years old, with the complaints of violation of nasal breathing on the right, constant nasal congestion of the right half of the nose, periodic congestion of the right ear and headache on the affected side, she was admitted for planned surgical treatment to ENT deprtment of Clinical Medical Center of A. I. Yevdokimov Moscow State University of Medicine and Dentistry for scheduled surgical treatment. The listed symptoms have been observed for about 10 years. Computed tomography showed a subtotal opacification of the right sphenoid sinus with spreading into the nasal cavity and nasopharynx. Preliminarily performed tumor biopsy with the following histological examination established an inverted papilloma. A transnasal endoscopic approach to the posterior sections of the nasal cavity was performed with the removal of the entire front and part of the lower walls of the right sphenoid sinus, removal of the tumor and wide dissection of the mucosa of the posterior sections of the nasal cavity and right sphenoid sinus in order to remove all possible loci of the primary focus. Planning of transnasal surgery begins with establishment a clinical diagnosis, confirmed in doubtful cases by the results of histological examination, analysis of the results of the necessary imaging method, and the selection of an adequate approach that contributes to complete dissection of the pathological formation and safe controlled surgery in aspects of blood loss and the injury of critical neurovascular structures within the least possible time period.

Keywords: inverted papilloma, sphenoid sinus, transnasal endoscopic approach.. 
For citation: Edzhe M. A., Ovchinnikov A. Yu., Shcherbakov A. Yu. Inverted papilloma of sphenoid sinus. Rossiiskaya otorinolaringologiya. 2020;19(1):105-109. https://doi.org/10.18692/1810-4800-2020-1-105-109

\section{Введение}

Инвертированная папиллома (ИП) или переходно-клеточная папиллома полости носа и околоносовых пазух - это доброкачественная опухоль, составляющая 0,4-7\% всех новообразований полости носа и околоносовых пазух, обладающая инвазивноподобным ростом по отношению к слизистой оболочке, поскольку гистологически отмечается не разрушение, а инвагинация покровного эпителия в подлежащую строму [1]. Разрушение костных стенок может быть обусловлено масс-эффектом при ее значительных размерах или трансформацией в плоскоклеточную карциному, что отмечается у 7-15\% больных [2]. Патогенез этого заболевания остается неясным, несмотря на то что у ряда пациентов установлена взаимосвязь заболевания с аллергией, хроническим синуситом и вирусной инфекцией [1-3].

Первичный очаг опухоли наиболее часто локализуется в слизистой оболочке решетчатого лабиринта (до 48\%) и верхнечелюстной пазухи (до 28\%). Реже первично поражаются другие околоносовые пазухи и структуры полости носа [4]. Первичное поражение клиновидной пазухи является редким и составляет около 7\% [5]. Из области первичного очага опухоль распространяется в соседние околоносовые пазухи и полость носа, вызывая соответствующие клинические симптомы. Изолированное поражение одной околоносовой пазухи является редким и отмечается в 5\% наблюдений [6].

Диагноз ИП устанавливается по данным осмотра, результатам применения методов визуализации - компьютерной томографии (КТ) и магнитно-резонансной томографии (МРТ), а также морфологического исследования биоптата. Проведение предварительной биопсии у пациентов с подозрением на ИП является обязательным для определения объема хирургического вмешательства. Что касается методов визуализации, то КТ и МРТ в этих случаях лишь дополняют друг друга. КТ является стандартом диагностики патологии околоносовых пазух, а МРТ - стандартом диагностики мягкотканых опухолей. В случае ИП на КТ определяются распространенность патологического процесса и целостность окружающих костных структур. При наличии гистологического заключения этого объема обследования оказывается достаточно, поскольку заподозрить инвазивный рост или злокачественное перерождение опухоли можно на основании КТ и только тогда дополнительно назначить МРТ. Что касается МРТ, то ряд авторов полагают, что это исследование позволяет установить первичную инвагинацию опухоли и предотвратить необходимость тотального удаления слизистой оболочки [1]. Другие авторы полагают, что при доказанной гистологически ИП достаточно выполнения только КТ, поскольку первичную инвагинацию опухоли можно установить только интраоперационно [8].

Одной из нерешенных хирургических задач, связанных с этим заболеванием, является высокая частота рецидивов после ее удаления, достигающая 50\% [7]. Объяснить рецидив опухоли только перерождением в злокачественный процесс не удается, поскольку уровень злокачественной трансформации гораздо ниже. Вероятным фактором рецидивирования является неполное удаление опухоли с сохранением остатков пораженной слизистой оболочки в труднодоступных анатомических зонах, особенно если речь идет о клиновидной пазухе, где неполная диссекция обусловлена близостью к критическим сосудистонервным структурам - зрительному нерву и сонной артерии [4].

В настоящее время стандартом хирургического лечения заболеваний полости носа и околоносовых пазух является эндоскопическая трансназальная хирургия, несмотря на то что открытые подходы сохраняют свою актуальность при невозможности осуществить все поставленные хирургические задачи эндоскопическим трансназальным способом $[8,9]$.

В этой связи важное значение приобретают планирование операции и выбор конкретного доступа.

\section{Пациенты и методы}

Больная Т., 57 лет, с жалобами на нарушение носового дыхания справа, постоянную заложенность правой половины носа, периодическую заложенность правого уха и головную боль на стороне поражения поступила на плановое хирургическое лечение в оториноларингологическое отделение Клинического медицинского центра МГМСУ им. А. И. Евдокимова. Перечисленные симптомы отмечает на протяжении 10 лет. Обследована в поликлинике по месту жительства. На КТ головы выявлено субтотальное затемнение правой основной пазухи с распространением в полость носа и носоглотку.

При осмотре слизистая оболочка полости носа справа резко отечна, при анемизации сокращается незначительно. Имеется искривление перегородки носа в средних отделах вправо в месте соединения хрящевой и костной частей. Обильный слизистый экссудат.

Поскольку при передней риноскопии опухоль не была визуализирована, проведено эндоскопическое исследование, при котором отмечено на- 

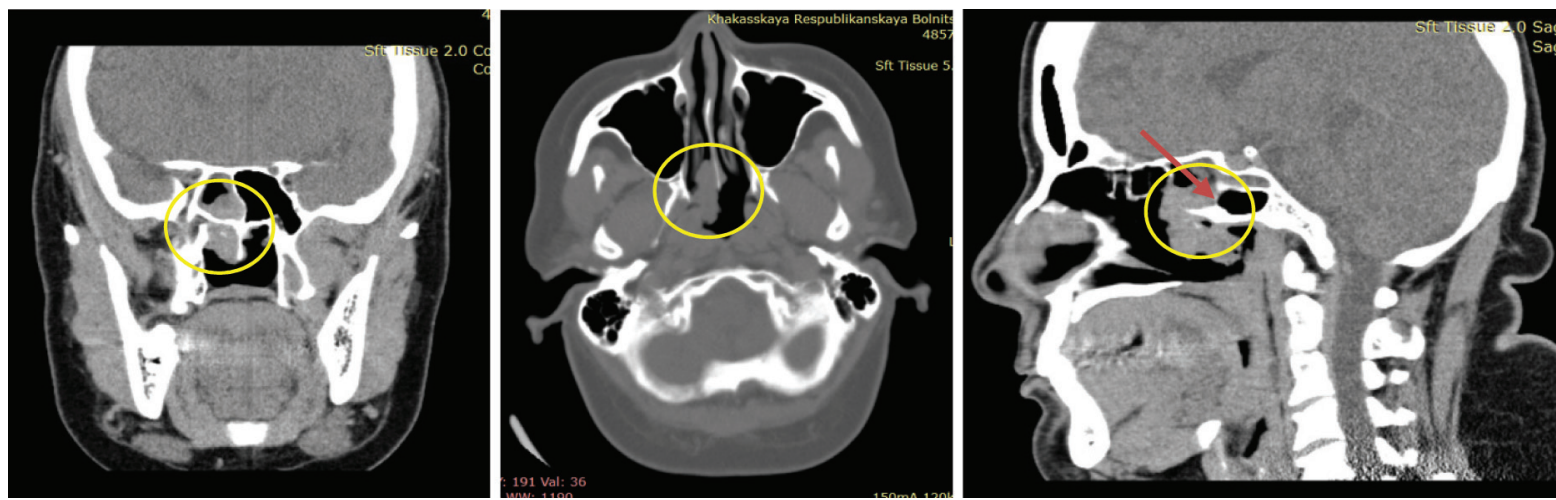

Рис. 1. КТ больной с инвертированной папилломой полости носа и правой клиновидной пазухи в трех проекциях. Отмечаются утолщение стенок пазухи, частичное разрушение ее передней стенки (красная стрелка), мягкотканое содержимое в передних отделах пазухи и задних отделах полости носа (желтый овал).

Fig. 1. Three CT scan projections of a patient with inverted papilloma of the nasal cavity and right sphenoid sinus. Thickening of the sinus walls, partial destruction of its anterior wall (red arrow), soft tissue content in the anterior part of sinus and posterior part of the nasal cavity are reveals (yellow oval).

личие мягкой бугристой ткани розового цвета в сфеноэтмоидальном пространстве справа.

Анализ КТ показал наличие мягкотканой опухоли, локализующейся в задних отделах полости носа и передних отделах правой клиновидной пазухи с расширением ее естественного соустья (рис. 1).

Предварительно выполнена биопсия опухоли с последующим гистологическим исследованием, в результате которого установлена инвертированная переходно-клеточная папиллома.

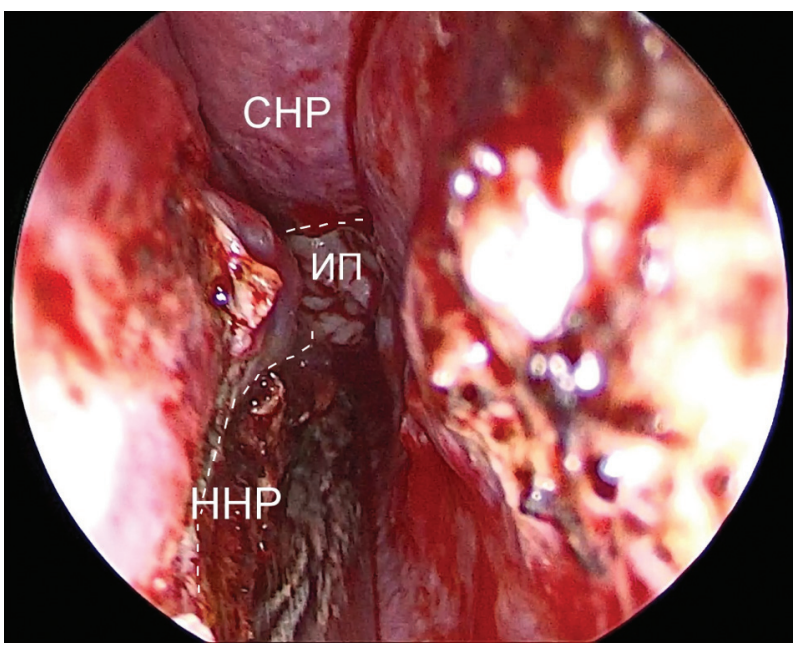

Рис. 2. Интраоперационная фотоэндоскопия. Резецированные задненижняя часть нижней носовой раковины и задний конец правой средней носовой раковины (пунктирная линия) и улучшенный обзор интраназальной части инвертированной папилломы. Условные обозначения: СНР - средняя носовая раковина, ННР - нижняя носовая раковина, ИП - инвертированная папиллома.

Fig. 2. Intraoperative photoendoscopy. A resected posterior and inferior part of the right inferior turbinate and posterior part of the right middle turbinate (dashed line) and an improved overview of the intranasal part of the inverted papilloma are presented. The legend: CHP - middle turbinate, ННР - inferior turbinate, ИП inverted papilloma.

\section{Результаты}

Учитывая результаты обследования, наиболее вероятными локусами первичной инвагинации в представленном наблюдении может являться слизистая оболочка как полости носа, покрывающая переднюю стенку КП, так и клиновидной пазухи в ее передних и нижних отделах. Поэтому в целях удаления всех возможных локусов первичного очага объем операции предусматривал удаление всей передней и части нижней стенок

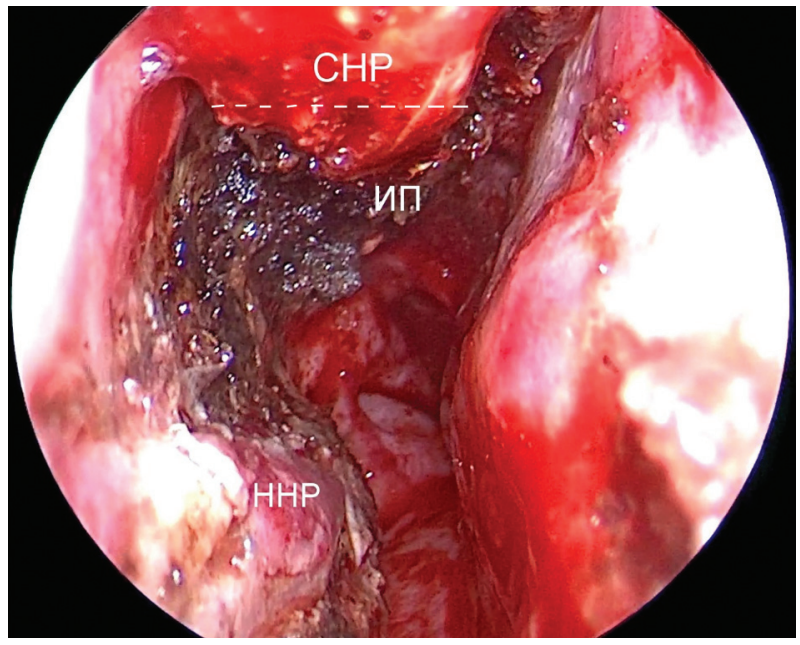

Рис. 3. Интраоперационная фотоэндоскопия. Заднелатеральные отделы полости носа. Зона небной кости. Проекция выхода ветвей клиновидно-небной артерии. Резекция нижней трети СНР (пунктирная линия). Условные обозначения: СНР - средняя носовая раковина, ННР - нижняя носовая раковина, ИП - инвертированная папиллома, КНА клиновидно-небная артерия, НК - небная кость.

Fig. 3. Intraoperative photoendoscopy. Postero-lateral part of nasal corridor. Palatal bone zone. The projection of sphenopalatine artery branches. Resected postero-inferior part of the right middle turbinate (dashed line). The legend: CHP - middle nasal turbinate, HНP - inferior nasal turbinate, ИП - inverted papilloma) КНА sphenopalatine artery, $\mathrm{HK}$ - palatal bone. 
правой клиновидной пазухи вместе со слизистой оболочкой, удаление интраназальной и внутрипазушной частей опухоли и широкую диссекцию слизистой оболочки правой клиновидной пазухи.

Для осуществления этого объема хирургии было необходимо определить широту доступа и объем резекции носовых раковин (нижней и средней), которые по данным эндоскопического осмотра и КТ были не вовлечены в опухолевый процесс, но находились в зоне доступа к опухоли. Эта проблема решалась интраоперационно.

Гипертрофию носовых раковин при опухолевом процессе можно объяснить вторичным воспалительным процессом. На первом этапе проведена латерализация нижней носовой раковины с резекцией ее задненижней части, а также с резекцией заднего конца СНР (рис. 2), что позволило полностью визуализировать интраназальную часть опухоли и расширить общий носовой ход для возможности работы в 4 руки: эндоскоп в левой руке хирурга, рабочий инструмент - в правой, аспиратор и управление системой ирригации у ассистента.

Опухоль представляла собой полиповидную массу, ярко-розового цвета, мягкой консистенции, которая достаточно легко удалялась.

Однако резекция задней трети СНР не позволила полностью визуализировать переднюю стенку КП, а угловую оптику было невозможно разместить выше интраназальной дрели в узком носовом коридоре, в связи с чем проведена диссекция нижней трети СНР (рис. 3). После этого стали обозримы зона небной кости, вся передняя стенка КП и задние решетчатые клетки.

В целях удаления слизистой оболочки в богато кровоснабжаемой зоне выхода ветвей клиновидно-небной артерии в полость носа была проведена верхнечелюстная синусотомия с удалением части медиальной стенки, ниже верхней трети крючковидного отростка, включающей соустье, до соединения с задней стенкой ВЧП. Из ВЧП удален воспалительный экссудат, не являющийся частью опухоли, выполнена подслизистая диссек- ция в области небной кости до обнажения ветвей клиновидно-небной артерии, которые были коагулированы биполярным пинцетом. Далее проведено удаление слизистой оболочки этой области и области передней стенки КП, удаление всей передней стенки, широкое открытие КП до нижней стенки и частичная ее диссекция при помощи интраназальной дрели. В этой зоне было отмечено кровотечение из палатовагинальной артерии, которая не выходит из клиновидно-небного отверстия общим стволом, а проходит в одноименном канале кзади от клиновидно-небного отверстия. Учитывая небольшой размер артерии, она была коагулирована биполярным пинцетом. Вдоль нижней стенки КП проходят видиев нерв и сопровождающая его артерия, которые могут повреждаться при широкой ее диссекции. Однако в представленном наблюдении этого не требовалось. После осуществления запланированного доступа к КП проведены удаление слизистой оболочки всей пазухи и коагуляция остатков в труднодоступных зонах углубления под турецким седлом и радиоволновым методом монополярным электродом-шариком.

Общее время операции составило 69 минут. Общая кровопотеря - 150 мл.

\section{Заключение}

Планирование трансназальной операции начинается с установления клинического диагноза, подтвержденного в сомнительных случаях результатами гистологического исследования, анализа результатов необходимого метода визуализации и выбора адекватного доступа, способствующего полноценному воздействию на патологическое образование и проведению безопасной контролируемой хирургии в аспектах кровопотери и повреждения критических сосудисто-нервных структур при наименьших из возможных временных затратах.

Авторы заявляют об отсутствии конфликта интересов.

\section{ЛИТЕРАТУРА/REFERENCES}

1. Lisan Q., Laccourreye O., Bonfils P. Sinonasal inverted papilloma: From diagnosis to treatment. European Annals of Otorhinolaryngology. Head and Neck Diseases. 2016;133;5:337-341. doi: 10.1016/j.anorl.2016.03.006.

2. Coutinho G., Marques J., Leal M., Spratley J., Fernandes M. S., Santos M. Surgical outcomes of sinonasal inverted papilloma: a 17 year review. Brazilian Journal of Otorhinolaryngology. 2019. doi: 10.1016/j.bjorl.2018.12.011.

3. Пестова Р. М., Савельева Е. Е., Азнабаева Л. Ф., Шарипов Р. А. Инвертированная папиллома на фоне двустороннего полипозного риносинусита с вязким муцином (клинический случай). Креативная хирургия и онкология. 2018;8;2:154-158 [Pestova R. M., Savel'eva E. E., Aznabaeva L. F., Sharipov R. A. Inverted papilloma on the background of bilateral polypous rhinosinusitis with viscous mucin (clinical case). Kreativnaya hirurgiya i onkologiya. 2018;8;2:154-158. (in Russ.)]. doi: 10.24060/2076-3093-2018-8-2-70-74.

4. Suh J. D., Ramakrishnan V. R., Thompson C. F., Woodworth B. A., Adappa N. D., Nayak J., Lee J. M., Lee J. T., Chiu A. G., Palmer J. N. Inverted papilloma of the sphenoid sinus: risk factors for disease recurrence. Laryngoscope. 2015;125;3:544-548. doi: 10.1002/lary.24929. 
5. Cui Y., Zhu Z. P., Sun Y., Wang Y. S. Sphenoid sinus inverted papilloma: a case report. Zhonghua Er Bi Yan Hou Tou Jing Wai Ke Za Zhi. 2016;7;51;11:857-858. doi: 10.3760/cma.j.issn.1673-0860.2016.11.013.

6. Lisan Q., Moya-Plana A., Bonfils P. Association of Krouse Classification for Sinonasal Inverted Papilloma With Recurrence: A Systematic Review and Meta-analysis. JAMA Otolaryngology-Head\&Neck Surgery. 2017;143;11:11041110. doi: 10.1001/jamaoto.2017.1686.

7. Sbrana M. F., Borges R. F., Pinna F. R., Brandão Neto D., Voegels R. L. Sinonasal inverted papilloma: rate of recurrence and malignant transformation in 44 operated patients. Brazilian Journal of Otorhinolaryngology. 2019. doi: 10.1016/j. bjorl.2019.07.00.

8. Yu Q. Q., Guan G., Zhang N. K., Zhang X. W., Jiang Y., Lian Y. Y., Liu T. T., Jiang X. D., Li N. Intranasal endoscopic prelacrimal recess approach for maxillary sinus inverted papilloma. European Archives Otorhinolaryngology. 2018;275;9:2297-2302. doi: 10.1007/s00405-018-5078-1.

9. Takahashi Y., Shoji F., Katori Y., Hidaka H., Noguchi N., Abe Y., Kakuta R. K., Suzuki T., Suzuki Y., Ohta N., Kakehata S., Okamoto Y. Endoscopic surgical management of sinonasal inverted papilloma extending to frontal sinuses. Otolaryngologia Polska. 2016;70;6:26-32. doi: 10.5604/01.3001.0009.3801.

\section{Информация об авторах}

Овчинников Андрей Юрьевич - профессор, доктор медицинских наук, заведующий кафедрой оториноларингологии, Московский государственный медико-стоматологический университет имени А. И. Евдокимова (127473, Россия, Москва, ул. Делегатская, д. 20, стр. 1); тел.: 7 (495) 609-67-00, e-mail: lorent1@mail.ru

ORCID: http://orcid.org/0000-0002-7262-1151

Эдже Майя Александровна - кандидат медицинских наук, профессор кафедры оториноларингологии, Московский государственный медико-стоматологический университет имени А. И. Евдокимова (127473, Россия, Москва, Делегатская ул., д. 20 , стр. 1); тел.: +7 (495) 609-67-00, e-mail: ayam75@mail.ru

ORCID: http://orcid.org/0000-0001-9509-9039

$\varangle$ Щербаков Андрей Юрьевич - кандидат медицинских наук, Московский государственный медико-стоматологический университет имени А. И. Евдокимова (127473, Россия, Москва, Делегатская ул., д. 20, стр. 1); тел. +7 (917) 598-30-60, е-mail: goalkeeper.89@mail.ru

ORCID: http://orcid.org/0000-0002-7490-8543

Information about the authors

Andrei Yu. Ovchinnikov - Professor, MD, Head of the Chair of Otorhinolaryngology, Evdokimov Moscow State University of Medicine and Dentistry (20, bld. 1, Delegatskaia str., Moscow, 127473, Russia); tel.: +7 (495) 609-67-00, e-mail: lorent1@mail.ru ORCID: http://orcid.org/0000-0002-7262-1151

Maiya A. Edzhe - MD Candidate, Professor of the Chair of Otorhinolaryngology, Evdokimov Moscow State University of Medicine and Dentistry (20, bld. 1, Delegatskaia str., Moscow, 127473, Russia); tel.: + 7 (495) 609-67-00, e-mail: ayam75@mail.ru ORCID: http://orcid.org/0000-0001-9509-9039

Andrei Yu. Shcherbakov - MD Candidate, Evdokimov Moscow State University of Medicine and Dentistry (20, bld. 1, Delegatskaia str., Moscow, 127473, Russia); tel.: +7 (917) 598-30-60, e-mail: goalkeeper.89@mail.ru ORCID: http://orcid.org/0000-0002-7490-8543 